\title{
Patterson Function from Low-Energy Electron Diffraction Measured Intensities and Structural Discrimination
}

\author{
Celia Rogero, Jose-Angel Martin-Gago and Pedro L. de Andres 7 \\ Instituto de Ciencia de Materiales (CSIC), Cantoblanco, 28049 Madrid, Spain
}

(Dated: March 13, 2021)

\begin{abstract}
Surface Patterson Functions have been derived by direct inversion of experimental Low-Energy Electron Diffraction I-V spectra measured at multiple incident angles. The direct inversion is computationally simple and can be used to discriminate between different structural models. $(1 \times 1)$ $\mathrm{YSi}_{2}$ epitaxial layers grown on $\mathrm{Si}(111)$ have been used to illustrate the analysis. We introduce a suitable R-factor for the Patterson Function to make the structural discrimination as objective as possible. From six competing models needed to complete the geometrical search, four could easily be discarded, achieving a very significant and useful reduction in the parameter space to be explored by standard dynamical LEED methods. The amount and quality of data needed for this analysis is discussed.
\end{abstract}

PACS numbers: $68.35 . \mathrm{Bs}, 07.79 .-\mathrm{v}, 61.14 . \mathrm{Hg}$

Low-Energy Electron Diffraction (LEED) takes advantage of strong interactions between LEED electrons and atoms to achieve highly sensitive and accurate surface structure determinations [1]. The prize to pay for that sensitivity based on a strong interaction is that simple first-order perturbation techniques are not enterily appropriated, and multiple-scattering is unavoidable to analyze the experimental data. Because multiple-scattering implies self-consistency [2], direct inversion of data to obtain the structure is quite difficult, and the most popular route to surface structural determination relies on a trialand-error procedure, that becomes too demanding when the parameter space to be explored is too big, or the structure cannot easily be guessed beforehand. Therefore, there is a genuine interest in developping and exploring direct inversion procedures, regardless of small practical difficulties or some limitations that might be found associated with them[3]. For LEED, an already succesful inversion technique based in ideas derived from holography exists 国, 5]. An useful alternative to holographic ideas, based in the Patterson Function (PF) concept, has also been proposed in the literature by Tong et al. [6. These two proposals are based in different physical concepts, but have in common that they yield useful information on the surface structure by directly performing on the experimental data a Fourier transform-like with an appropriate kernel. The main desired effect of this mathematical operation is to filter out high order scattering terms. Out of necessity, this is only an approximate procedure, but previous experience with holography, iterative methods based in maximum entropy [7], or even quasi-dynamical methods [8] have shown that multiplescattering is not necessarily a roadblock.

In this paper we explore the PF idea, and by considering a practical example based in experimental LEED-IV data measured at different energies and incident angles we show how useful these ideas can become when they are used to complete a real surface structure determi- nation. The strategy to defeat the limitations imposed by multiple-scattering is to collect data simultenously at different energies and incident angles. This results in overdetermination of the inverted data, where coincidences in features not directly related to the underlaying geometrical structure decrease as the data base increase [9]. Therefore, the effort to get the structure shifts from the trial-and-error search to the measurement part, which is not a real burden taking into account modern LEED data acquisition techniques.

The surface Patterson function (PF) can be computed by an appropriate phase sum of the measured intensities [6]:

$$
P(\vec{r})=\left|\sum_{\vec{k}_{i}} \sum_{\vec{g}_{\|}} \int I\left(\vec{k}_{i}, \vec{q}\right) e^{i \vec{q} \vec{r}} d q_{\perp}\right|^{2}
$$

where $\vec{k}_{i}$ is the wavevector corresponding to incoming electrons, $\vec{q}$ represents the momentum transfer for outgoing electrons detected in the LEED screen $\left(\vec{k}_{f}=\vec{k}_{i}+\vec{q}\right)$, and $\vec{g}_{\|}$labels the different beams. Simple real-space scattering models analyzed by Tong et al.[6] help us to understand why the multi-energy and multi-angle approach can reduce spurious features appearing in the stationary phase condition implied in Eq. (1). The PF yields maxima at positions related to interatomic distances: we shall find that these can be used to discriminate reasonable from unreasonable structural candidates.

To better understand how useful these ideas might be for real structural determination we take advantage of the experimental LEED I(V) curves we have recently measured at normal and non-normal incidence for $(1 \times 1)$ $\mathrm{YSi}_{2}$ layers grown on $\mathrm{Si}(111)$ substrates. These measurements have been used to perform a standard I(V) study of the structure, where the best correlation was found at a Pendry R-factor 10 of $R_{P}=0.21$, which is accepted to be a low-enough value to credit the structural model. Details related to this model have been 
published elsewhere [1], but the structural model is presented in Fig. (1A) for reference. Basically, the surface is made of $\mathrm{Si}(111)$ planes $180^{\circ}$ rotated around the $\mathrm{Si}(111)$ surface normal, with Y atoms located below the surface on $\mathrm{T}_{4}$ sites (w.r.t the $\mathrm{Si}(111)$ substrate). The small relaxations between layers included in the model are not crucial for the analysis presented in this paper, because the spots on the PF already have an intrinsic width on the same order or bigger than the values associated to the pure geometrical surface relaxations.

Experimental LEED I(V) curves were recorded at normal and non-normal incidence. The latter was achieved by moving the surface normal in a plane defined by the surface normal and the $[\overline{1} 2 \overline{1}]$ surface direction. Measurements at $\theta=0^{\circ}, 4^{\circ}, 9^{\circ}$ and $14^{\circ}$ where taken for the same azimuthal angle, $\phi .19$ beams (6 of which are not symmetry-related) where measured from 50 to $450 \mathrm{eV}$, except for normal incidence where only 18 beams could be collected (the specular beam was blocked by the electron gun). Therefore, the total energy range for independent data is more than $8000 \mathrm{eV}$. The experiment was performed at room temperature: in the standard LEED analysis it was found that fine tuning of isotropic vibrations associated to different layers could make a strong impact on the $\mathrm{R}_{P}$ value, decreasing its value from around 0.4 for r.m.s. displacements taken from bulk values, to around 0.2 for the final optimized surface values 11. At the moment these effects are not taken into account in Eq. (11), but we anticipate their main effect is to broaden the PF increasing the values of the correlation factor we have found below.

The PF gives the interatomic distances between all atomic pairs in the structure. To simplify the discussion and make a better representation of our results, we have chosen the [101] cross-sectional plane, but the same conclusions would be obtained using a different plane. Fig. (11B) shows the interatomic distances for one unit cell calculated from the structure in Fig. (1A): stars correspond to positions where a perfect stationary phase condition would produce spots in the PF from I-V spectra. The corresponding atomic pairs are indicated next to the stars, using the same labelling as in Fig. (1A). This is a mere geometrical PF, and it is the best result that could ever be recovered for a given structure. Therefore, we compute correlations between experimental PF obtained from Eq. (11) using all measured diffracted intensities and geometrical PF corresponding to different trial structures. As shown in Fig. (2), the experimental PF is made of spots with a certain width derived from factors like the limited domain of integration, atomic vibrations, defects, etc.

A visual comparison between the stars in Fig. (11B) and the spots in Fig. (2) induces us to introduce a correlation factor between PF's to facilitate their comparison. The new correlation factor might be defined in different ways, but to demonstrate the concept we shall here introduce
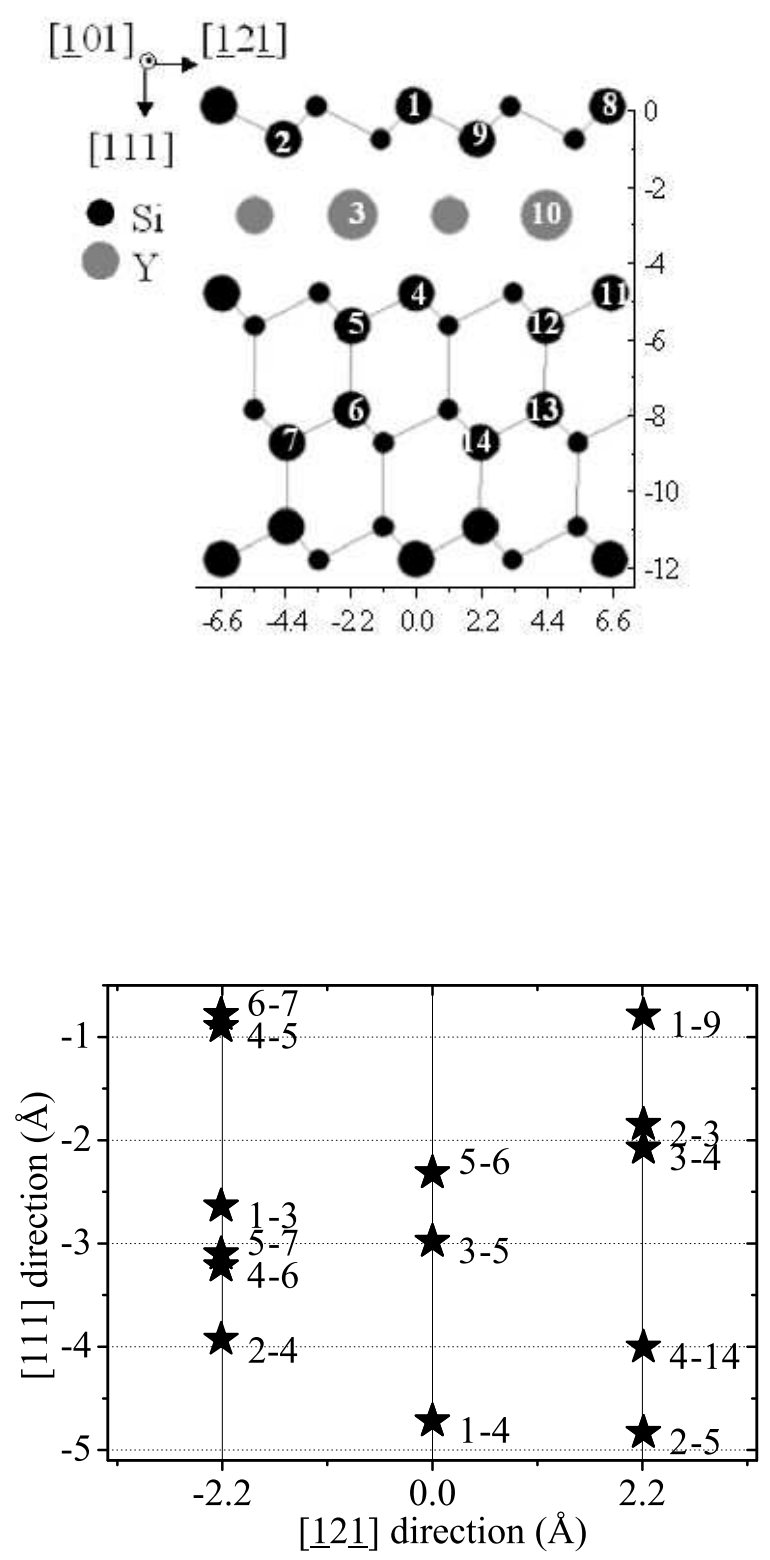

FIG. 1: (A) Upper pannel: Schematic positions and labelling of atoms in $\mathrm{YSi}_{2} / \mathrm{Si}(111)$. The geometry corresponds to the best fit determined to experimental LEED data measured on the same system 11] (B) Lower pannel gives the geometrical Patterson Function related to structure in upper pannel. 


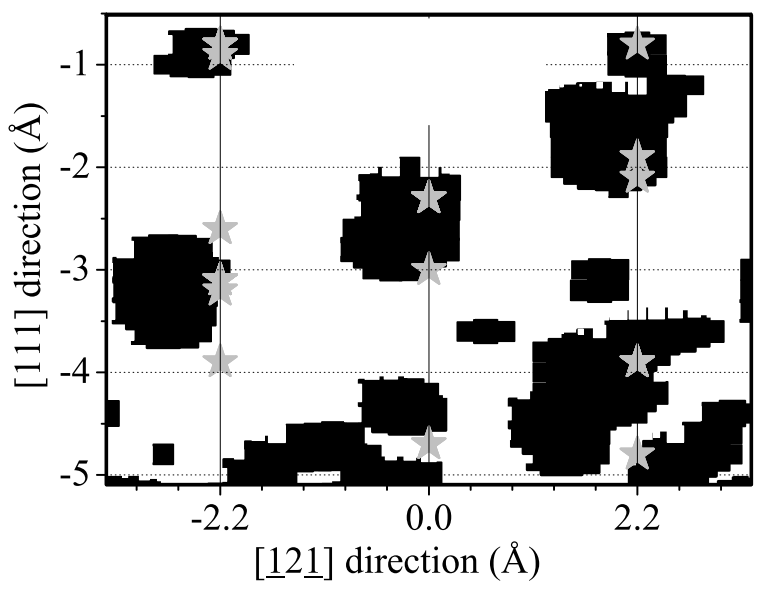

FIG. 2: PF from Eq. (11), and the experimental I(V) curves measured for 19 beams at four different incident angles.

the simplest one, to keep the discussion as independent as possible from particular details of this definition:

$$
R_{P F}=\frac{S_{\text {spots }}}{S_{P F}} \frac{\sum D}{N_{g e o}}
$$

where $D$ is the distance between stars corresponding to a given structure and the center of the closest experimental spot around it, $N_{\text {geo }}$ is the number of interatomic distances considered, $S_{\text {spots }}$ is the area covered by the spots, and $S_{P F}$ is the total area considered for the analysis. The factor $\frac{S_{s p o t s}}{S_{P F}}$ gives the probability to find a spot in the considered area, whilst the factor $\frac{\sum D}{N_{\text {geo }}}$ measures how far are the experimental spots from the corresponding stars. In order to preserve the meaning of this particular $R_{P F}$, the factor $\frac{S_{s p o t s}}{S_{P F}}$ should not be too close to 1 (e.g., spots covering the whole surface) or to 0 (e.g., too few spots). By constructing model geometries at random, we estimate typical values for $R_{P F}$ to be around 0.25-0.20 when the structures are not correlated.

The structural analysis for $\mathrm{YSi}_{2}$ epitaxially grown on $\mathrm{Si}(111)$ requires considering six different models for different stackings of the atomic layers. In our previous analysis of this structure 11 , a detailed $\mathrm{R}_{P}$ analysis covering different values for all the considered parameters has been performed. This extensive search reflects the sensitivity and highly non-linear dependence of intensities with structural parameters in multiple-scattering calculations 12]. This is not the case for PF, where a stationary phase condition based in two first-order scattering events is sought. Therefore, the advantage of computing correlations between experimental PF and different models is double: (i) there is no need to run costly multiple-scattering calculations for the whole parameter space, and (ii) because the analysis is based in first- order scattering events, the $\mathrm{R}_{P F}$ is expected to behave smoother as a function of the involved parameters, and less local minima are expected. Although, on the other hand, a smoother variation may result in less sensitivity to different structures. Correlations between the experimental PF and the different models considered in the previous structural analysis are given in table $\mathbb{\text { If }}$. To complement $\mathrm{R}_{P F}$, we give next to it a number counting the percentage of coincidences between the stars and the PF spots. This has been defined as the number of stars which are inside the region in black in the figures. This number can be helpful to discriminate between models, as can been seen in Table I, but it has not been included in the $\mathrm{R}_{P F}$ definition because it depends on the width of the spots (a function of several factors, e.g. the pure thermal vibration of atoms is expected to contribute an intrinsic width in the order of several tenths of Angstroms).

From entry named Experiment in table I we can inmediately reduce the available six models to two (i.e., a $66 \%$ saving has already been achieved by simply Fouriertransforming the experimental data). Differences in $R_{P F}$ between model $\mathrm{B}$ and $\mathrm{F}$, however, are not big enough to discriminate between them. In fact, the lowest $R_{P F}$ corresponds to model $\mathrm{F}$, whilst the best fit based in $R_{P}$ corresponds to the second lowest $R_{P F^{-v a l u e}}$ model $\mathrm{B}$. Notice that in both cases, the percentage of coincidences are among the highest.

To understand the origin of this different ordering in models based in PF analysis, we test the consistency in the definition of $R_{P F}$ by replacing the experimental $\mathrm{I}(\mathrm{V})$ curves by theoretical ones corresponding to the structure B. Results shown in table 1 under the column named Theory (19 beams) show that the definition for the Rfactor is internally consistent and now the best model is clearly the correct one. Therefore, the question arises of what is the effect on the PF of exchanging sets of $\mathrm{I}(\mathrm{V})$ curves (i.e., experiment and theory) that differ themselves by $R_{P}=0.21$. We notice that multiple-scattering cannot be the source for the discrepancy as both, the experimental and the theoretical intensities include all the multiple-scattering relevant for the problem: more likely this is due to residual factors (like anisotropic vibrations of atoms near the surface, defects, etc) or the size of the integration domain itself. Therefore, we turn to a visual comparison of Fig. (2) and Fig. (3A). Both PF's have a similar probability to find a spot: $\frac{S_{\text {spots }}}{S_{P F}}=0.40$ for experiment and $\frac{S_{s p o t s}}{S_{P F}}=0.39$ for theory, but widths of individual spots are too wide to obtain an accurate correlation with stars related to models B and F. Due to spurious features, both models become undistinguishable: this is not too surprising as the two models in question are very similar.

Two simple ways to narrow these widths come to mind. From an experimental point of view, measurements made at low temperatures or recorded on samples as perfect as 
TABLE I: $R_{P F}$ and coincidences for different stacking models. Results are shown for the experimental data and two theoretical simulations (19 and 91 beams). Coincidences give the percentage of geometrical stars inside experimental or theoretical PFspots.

\begin{tabular}{|c|c|c|c|c|c|c|c|}
\hline \multirow[t]{2}{*}{ Code } & \multirow[t]{2}{*}{ Structural Model } & \multicolumn{2}{|c|}{ Experiment } & \multicolumn{2}{|c|}{ Theory (19 beams) } & \multicolumn{2}{|c|}{ Theory (91 beams) } \\
\hline & & $\mathrm{R}_{P F}$ & (\% Coinc.) & $\mathrm{R}_{P F}$ & (\% Coinc.) & $\mathrm{R}_{P F}$ & (\% Coinc.) \\
\hline $\mathrm{A}$ & $\mathrm{T}_{4}$ Rotated $0^{\circ}$ & 0.159 & 40 & 0.114 & 60 & 0.113 & 67 \\
\hline $\mathrm{B}$ & $\mathrm{T}_{4}$ Rotated $180^{\circ}$ & 0.140 & 50 & 0.069 & 88 & 0.026 & 88 \\
\hline $\mathrm{C}$ & $\mathrm{H}_{3}$ Rotated $0^{\circ}$ & 0.175 & 40 & 0.122 & 60 & 0.146 & 53 \\
\hline $\mathrm{D}$ & $\mathrm{H}_{3}$ Rotated $0^{\circ}$ & 0.150 & 47 & 0.123 & 59 & 0.060 & 65 \\
\hline $\mathrm{E}$ & Atop Rotated $0^{\circ}$ & 0.161 & 50 & 0.088 & 73 & 0.107 & 86 \\
\hline $\mathrm{F}$ & Atop Rotated $180^{\circ}$ & 0.136 & 67 & 0.083 & 73 & 0.030 & 80 \\
\hline $\mathrm{G}$ & random model & 0.250 & 13 & 0.250 & 13 & 0.180 & 6 \\
\hline
\end{tabular}
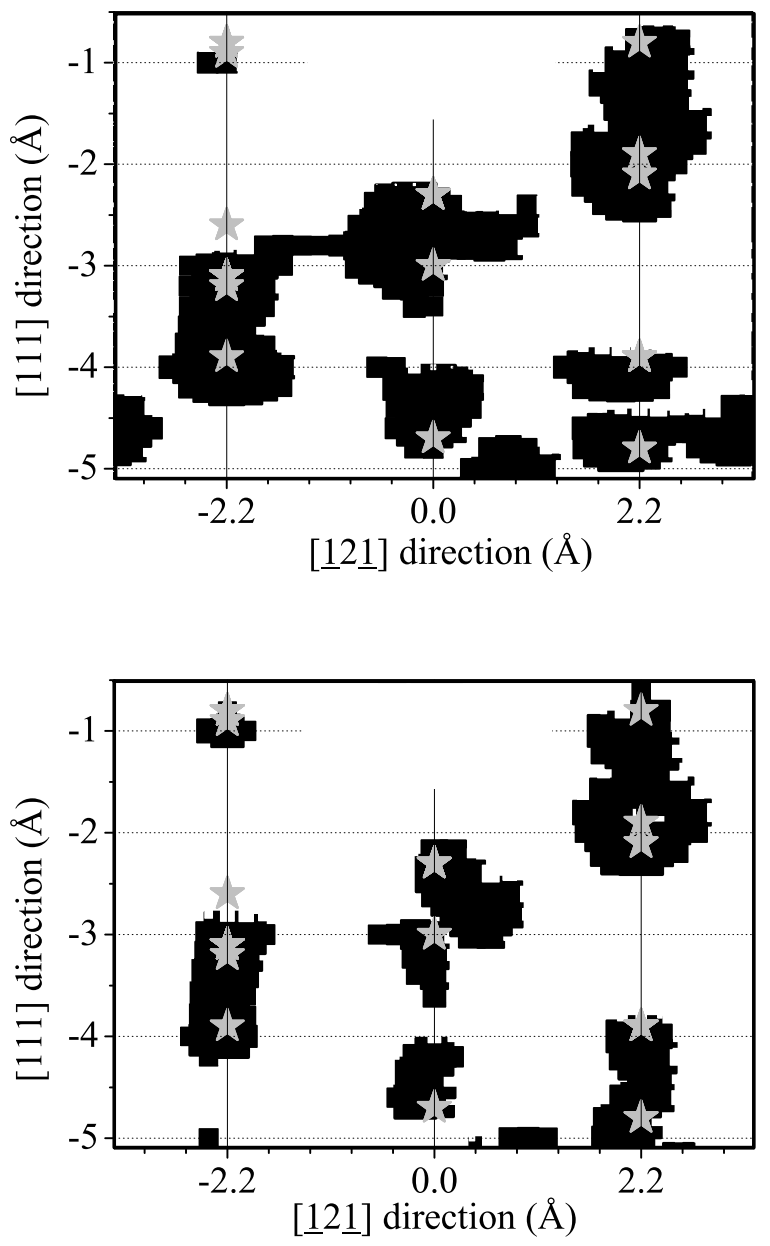

FIG. 3: (A) Upper pannel shows the PF obtained from Eq. (1), and the theoretical I(V) curves for 19 beams at four different incident angles computed for geometry in Fig. (1). (B) Lower pannel shows the PF obtained from Eq. (1), and the theoretical I(V) curves measured for 91 beams at four different incident angles. possible are needed. On the other hand, the stationary phase condition implied in Eq. 11 requires covering as much volume as possible in the Fourier-transform domain (i.e., measuring as many beams at different angles for as many energies as possible).

Our experimental setup puts some limits on the number of beams we can measure, and restrict us to room temperature. We comment in passing that analyzing a $1 \times 1$ put us in the worst possible case for PF analysis. For the same energy range, any other superstructure will provide extra beams to be measured. Furthermore, the $1 \times 1$ usually would contribute less stars than a system with a surface superstructure, where different pair distances are involved. Finally, beams in the $1 \times 1$ mix surface and bulk information, a disadvantage that the fractional beams corresponding to a genuine surface superstructure do not present.

Therefore, we explore the second requirement by substituting our experimental I(V) curves by theoretical I(V) curves computed for model B for all the possible exiting beams (i.e., 91 beams in total). Indeed, comparison between Figs. (3 A) and (3 B) demostrates that the widths of the PF spots narrow when the data base is increased: the probability to find a spot has been reduced to $\frac{S_{s p o t s}}{S_{P F}}=0.25$, nearly a factor of two w.r.t. the theory or the experiment based in 19 beams. Clearly, if we can measure more beams we could obtain more accurate correlation between stars and PF-spots.

In conclusion, we have found that the PF concept allows us to significatively cut the parameter space to be explored in a real structure determination based in LEED data measured at different angles and energies. The extra burden of having to measure more beams and the requirement for liquid Nitrogen cooling temperature should not be excesive for modern LEED equipment. The advantage is significative. The analysis based in the PF is very fast compared with the standard procedure, as it mainly implies Fourier-transforming the measured data. Inside the restricted parameter space determined by the PF analysis, it seems appropriate to run multiple-scattering cal- 
culations. This is certainly more costly than the simple integral involved in PF but it might be worthwhile: its intrinsic non-linear dependence with the structure, and the presence of higher order correlations, will help to obtain accurate and trusty values for the final parameters of the model.

Work supported by the Spanish Ministry of Science and Technology (PB98-524).

* Electronic address: pedro@mailaps.org

[1] M. V. Hove, W. Weinberg, and C. Chang, Low-Energy Electron Diffraction (Springer-Verlag, Berlin, 1986).

[2] J. Pendry, Low-Energy Electron Diffraction (Academic,
London, 1974).

[3] D. Adams and U. Landman, Phys. Rev. B 15, 3775 (1977).

[4] D. Saldin and P. de Andres, Phys. Rev. Lett. 64, 1270 (1990).

[5] K. Heinz, A. Seubert, and D. Saldin, J. Phys.: Condens. Matter 13, 10647 (2001).

[6] H. Wu and S. Tong, Phys. Rev. Lett. 87, 036101 (2001).

[7] D. Saldin, A. Seubert, and K. Heinz, Phys. Rev. Lett. 88, 115507 (2001).

[8] N. Bickel and K. Heinz, Surf. Sci. 163, 435 (1985).

[9] P. de Andres, Surf. Sci. 269-270, 1 (1992).

[10] J. Pendry, J. Phys. C 13, 937 (1980).

11] C. Rogero, C. Polop, L. Magaud, and al., Phys. Rev. B (in press) (2002).

[12] P. de Andres and J. Verges, Phys. Rev. B 59, 3086 (1999). 